\section{Sterbehilfe aus der Sicht der jüdischen Medizinethik}

\author{
Y. Nordmann
}

jüdischen Religion keine klare Trennlinie zwischen Ethik und Gesetz existiert. Vereinfacht gesagt: Das Gesetz ist die Ethik, die Ethik ist das Gesetz. Es erscheint so unumgänglich, das jüdische Gesetz zu kennen, will man die jüdische Medizinethik verstehen: Die jüdische Religion basiert auf zwei Fundamenten: Dem schriftlichen Gesetz und der mündlichen Überlieferung. Das schriftliche Gesetz, welches im allgemeinen Sprachgebrauch auch als Bibel bezeichnet wird, setzt sich aus drei Teilen zusammen, nämlich der Tora, den Neviim und den Ketuvim, wobei die Tora sicherlich die grösste Bedeutung einnimmt. Sie ist die Grundlage des jüdischen Glaubens und beinhaltet in fünf Büchern die göttliche Lehre, die Moses am Berge Sinai erhalten hat. Die jüdische Lehre besteht aber nicht nur aus dem schriftlichen Gesetz. Vielmehr kommt die mündliche Überlieferung hinzu, ohne die vieles des schriftlichen Gesetzes unverständlich bliebe. Die mündliche Überlieferung wurde Moses und dem jüdischen Volk am Berge Sinai zur gleichen Zeit wie das schriftliche Gesetz von Gott übergeben. In einer langen, beständigen Kette der Tradition blieb sie bis in die heutige Zeit erhalten. Im 2. Jh. n. Chr. wurde damit begonnen, die Lehren der mündlichen Überlieferung in einem Werk zusammenzustellen, welches schliesslich als sogenannter Talmud weit über die jüdische Religion hinaus Bekanntheit erlangen sollte. Der Talmud spielt in der jüdischen Tradition eine äusserst wichtige Rolle, stellt er doch eine Art Enzyklopädie des gesammelten jüdischen Wissens dar.

\section{Was ist unter dem Begriff «Halacha» zu verstehen?}

Jegliche Beschäftigung mit Problemen im Rahmen des jüdischen Gesetzes, der sogenannten Halacha, geht nur über ein Studium dieser Quellen, also Tora und Talmud sowie deren Kommentare. Es ist somit gemäss der Denkweise der jüdischen Medizinethik nicht möglich, moralische Probleme der Medizin auf der Basis von persönlichen Gefühlen und Wertvorstellungen zu lösen, sondern es bedarf der eingehenden halachischen Analyse, durchgeführt durch eine rabbinische Autorität, deren Entscheidung dann zu respektieren ist. Dabei reicht eine schlichte Vertrautheit des Rabbiners mit den halachischen Quellen nicht aus. Vielmehr müssen in jedem spezifischen Fall die jeweiligen Umstände in die Überlegungen miteinbezogen werden, es muss also von Fall zu Fall entschieden werden. Ein weiterer, ganz zentraler Punkt muss an dieser Stelle angefügt werden: Die jüdische Religion wird nicht durch eine über allem stehende Autorität geleitet, wie dies beispielsweise im Katholizismus der Fall ist. Vielmehr existieren im Judentum verschiedene sogenannte "Poskim», was auf Deutsch mit «Entscheidungsträger» übersetzt werden könnte. Bei diesen Entscheidungsträgern handelt es sich um äusserst gelehrte, hochqualifizierte Rabbiner, die sich durch ihre Entscheidungen und Responsen weltweite Anerkennung verschafft haben. Ein Entscheidungsträger wird nicht von irgendeiner Instanz

Korrespondenz:

Dr. med. Yves Nordmann

Mutschellenstrasse 55

CH-8038 Zürich 
in diese Funktion gewählt, er «erhält» diese Rolle vielmehr durch persönliche und fachliche Autorität, welche sich vor allem dadurch äussert, dass er Anfragen aus der ganzen Welt zugestellt bekommt. Die meisten Gemeinderabbiner und der grösste Teil der orthodoxen jüdischen Weltbevölkerung akzeptieren die Entscheidungen einer solchen rabbinischen Kapazität. In der heutigen Generation sind es u.a. Rav Joseph Elijaschiv und - speziell für medizinische Fragen - Rav Elieser Jehuda Waldenberg, beide in Israel, welche die Rolle von "Poskim» erhalten haben und ausüben. Nun kann es durchaus vorkommen, dass verschiedene "Poskim» in gleichen Sachfragen unterschiedliche Entscheidungen treffen, obschon die gleiche Quelle, nämlich die Tora, zur Analyse des Problems benutzt wurde. Wie kann dies erklärt werden? Die Worte der Tora selbst sind, wie vorher angetönt, unabänderbar; ihre Interpretation unterliegt aber dem menschlichen Intellekt, wie in der Bibel (Deuteronomium, 30:12) geschrieben steht: "lo baschamaim hi», sie, gemeint ist die Tora, ist nicht im Himmel. Es liegt in den Händen des Menschen, die Tora zu interpretieren und praktisch anzuwenden. Die jüdische Lehre lässt es im übrigen durchaus $\mathrm{zu}$, dass zwei widersprüchliche Schlussfolgerungen von verschiedenen Autoritäten aus derselben Quelle abgeleitet werden. Welche ist nun korrekt? Der Talmud selbst gibt uns die Antwort: Beide! Wie geschrieben steht (Gittin, 6b): «Dies und das sind die Worte des lebendigen Gottes." Es muss aber an dieser Stelle betont werden, dass im jüdischen Glauben auch verschiedene, ganz zentrale Grundprinzipien existieren, die allgemein unumstritten sind, da sie explizit in der Tora erwähnt werden. Gerade an der Problematik der Sterbehilfe lassen sich einige dieser Prinzipien besonders gut veranschaulichen.

\section{Grundaspekte der Sterbehilfe aus jüdischer Sicht}

\section{Tötungsverbot}

Es ist gemäss der Tora generell verboten, einen Menschen umzubringen. Die einzige Situation, in der dies doch erlaubt sein könnte, tritt in Notwehrsituationen ein: Wenn es sich beim Umzubringenden um einen potentiellen Mörder handelt und nur durch dessen Tötung der eigene Tod oder der eines anderen Unschuldigen verhindert werden kann, so ist dies gerechtfertigt, wenn keine andere Möglichkeit besteht, den potentiellen Mörder von seiner Tat abzuhalten. Das Umbringen eines Menschen aber, der nicht eine unmittelbare Lebensbedrohung für andere darstellt, gilt als Mord, ob es sich dabei nun um ein Neugeborenes, einen gesunden Erwachsenen oder einen Sterbenden handelt. Unter das Tötungsverbot fällt explizit auch das Verbot der Selbsttötung [1]. Der Suizid ist selbst unter der Absicht verboten, einem anderen Menschen durch diese Tat das Leben zu retten.

\section{Leben als "Leihgabe»}

Der Mensch besitzt nicht ein absolutes Anrecht auf seinen Körper. Gott hat jedem Menschen einen Körper und eine Seele für eine bestimmte Zeit zur Verfügung gestellt, und jeder Mensch ist verantwortlich dafür, zu dieser "Leihgabe» Sorge zu tragen [2]. Aus jüdischer Sicht ist es deshalb grundsätzlich nicht erlaubt, frei über seinen Körper zu verfügen, sich willentlich Verletzungen zuzuführen oder Selbstmord zu begehen.

\section{Unendlicher Wert menschlichen Lebens}

Einer der zentralen Grundsätze des jüdischen Glaubens liegt in der Auffassung, dass menschliches Leben Heiligkeit sowie absoluten, unantastbaren und unendlichen Wert besitzt. Rabbiner Lord Immanuel Jakobovits (1921-1999), ehemaliger Oberrabbiner von Grossbritannien und Experte auf dem Gebiet der jüdischen Medizinethik, hat eine treffende Beschreibung dieses Prinzips formuliert:

"[...] the value of human life is infinite and beyond measure, so that any part of life - even if only an hour or a second - is of precisely the same worth as seventy years of it, just as any fraction of infinity, being indivisible, remains infinite. Accordingly, to kill a decrepit patient approaching death constitutes exactly the same crime of murder as to kill a young, healthy person who may still have many decades to live." [3]

Hier wird die Grundhaltung der jüdischen Medizinethik gegenüber der Sterbehilfe bereits sehr klar deutlich: Der unendliche Wert menschlichen Lebens stellt aus jüdischer Sicht eine unabdingbare Voraussetzung für eine moralisch hochstehende Gesellschaft dar. Da auch der Bruchteil einer Unendlichkeit eine Unendlichkeit ist, besitzt jeder Moment des menschlichen Lebens den gleichen, unendlichen Wert. Würde eine Person, die noch wenige Tage oder Stunden zu leben hat, weniger Wert besitzen, als ein Mensch, der aller Voraussicht nach noch 70 Jahre leben wird, so würde der unendliche Wert menschlichen Lebens seinen absoluten Charakter verlieren und relativ werden. Er würde relativ zur Lebenserwartung, relativ zum Gesundheitszustand oder relativ zum Nutzwert für die Gesellschaft eingestuft werden. Beliebige, willkürlich gewählte Kriterien könnten herangezogen werden, um über den "Wert" oder "Unwert" eines menschlichen Lebens zu befinden. Es käme zu einer Einteilung von Menschen in höhere und tiefere Klassen, in Menschen, die einen berechtigteren Anspruch auf Leben haben und solche, die keinen Anspruch auf Leben mehr besitzen. Es bestünde die Gefahr, in ein ähnliches Wertsystem abzugleiten, wie es noch vor kurzer Zeit in einem der dunkelsten Kapitel der Weltgeschichte Geltung beanspruchte. Es darf so nicht erstaunen, dass gerade die jüdische Religion der Problematik der Sterbehilfe gegenüber einen ausgesprochen sensibilisierten Zugang hat. Der Philosoph Jeshajahu Leibovitz drückt dies in eindrücklichen Worten aus [4]: «Die Frage, ob ein Leben lebenswert ist, darf es nicht geben." 


\section{Definition und Status des Sterbenden aus jüdischer Sicht}

Nach Vorstellung dieser Grundprinzipen im Zusammenhang mit der Sterbehilfe aus jüdischer Sicht, soll im folgenden der Frage nachgegangen werden, wie der Sterbende in klassischen halachischen Quellen definiert wird und welchen Status er dabei besitzt. Der halachische Fachausdruck für den Sterbenden, wie er im Talmud und von den rabbinischen Autoritäten verwendet wird, lautet "goses", was einen Menschen bezeichnet, der im unmittelbaren Angesichte des Todes steht. Eine exaktere Definition dieses Terminus erweist sich als schwierig, auch wenn verschiedene Gelehrte den Versuch einer genaueren Bestimmung gewagt haben: Rabbi Mosche Isserles (Rema, 16. Jh.) beispielsweise sieht den "goses" als einen «Patienten, der dem Tod so nahe ist, dass er Schleim hochwürgt, da er derartige Schwierigkeiten hat, zu atmen" (Kommentar zu Even Haeser, 121:7, und Choschen Mischpat, 211:2; eigene Übersetzung). Für Rav Bleich, einen zeitgenössischen Rabbiner, ist diese «klinische Definition" für unsere Zeit ungenügend. Für ihn ist schlicht derjenige Mensch ein "goses", der mit Sicherheit und unter allen Umständen (auch mit der Hilfe von Medikamenten) nicht mehr als noch 72 Stunden zu leben hat [5]. Während über die Definition des Sterbenden also einige Unklarheit herrscht, wird bereits in frühen Quellen der Status des Sterbenden sehr eindeutig bestimmt. Im Talmudtraktat Semachot, 1:1-4, heisst es beispielsweise (eigene Übersetzung): "Jemand, der am Sterben ist, wird in jeder Beziehung wie ein Lebender betrachtet. Man soll ihm [dem Sterbenden] weder den Kiefer binden, noch seine Öffnungen zustopfen [...] Man darf ihn nicht bewegen [...] Man darf die Augen einer sterbenden Person nicht schliessen. Derjenige, der ihn [den Sterbenden] berührt und bewegt, der vergiesst Blut, wie Rabbi Meir zu sagen pflegte: Dies kann mit einer schwachen Flamme verglichen werden; sobald eine Person sie berührt, erlöscht sie. So auch hier: Wer die Augen eines Sterbenden schliesst, wird so angesehen, als ob er ihm seine Seele genommen hätte [...].» Aus dieser Quelle geht sehr eindrücklich hervor, wie streng die Gesetze im Zusammenhang mit dem Sterbenden aus jüdischer Sicht gefasst sind: Dem obersten Prinzip der Heiligkeit menschlichen Lebens folgend, ist es verboten, den Tod eines Sterbenden zu beschleunigen. Aus diesem Grund darf ein Sterbender nicht einmal berührt und bewegt werden (eine Ausnahme hierzu bilden gemäss den rabbinischen Autoritäten medizinische oder pflegerische Massnahmen, welche am Patienten notwendig erscheinen). Auch wenn sich bis anhin gezeigt hat, wie streng die Gesetze im Zusammenhang mit dem Sterbenden aus halachischer Sicht gefasst sind, so finden sich dennoch durchaus einige Quellen in jüdischen Schriften, die zur Verteidigung der passiven Sterbehilfe herangezogen werden können. Es gilt zu betonen, dass sich die folgenden Ausführungen immer nur auf einen sogenannten "goses" beziehen, also einen Sterbenden, der in unmittelbarem Angesichte des Todes steht. Eine erste wichtige Quelle findet sich bei Rabbi Jehuda ben Samuel, der um das Jahr 1200 n. Chr. in seinem «Sefer Hachassidim» (Buch der Frommen) schrieb: «[...] wenn eine Person am Sterben ist, und in der Nähe ihres Hauses fällt jemand Holz, so dass die Seele [des Sterbenden] nicht entfliehen kann, so soll jemand den [Holz-]Fäller von dort vertreiben.» [6] Basierend auf dieser Aussage äusserte sich Rabbi Mosche Isserles (16. Jh.) wie folgt: "[...] wenn irgendetwas vorhanden ist, was die Seele daran hindert, zu entfliehen, wie etwa ein klopfendes Geräusch in der Nähe des Hauses des Patienten [...], und dies hindert die Seele daran, $\mathrm{zu}$ entfliehen, so ist es erlaubt, dieses Hindernis zu entfernen, da auf diese Weise keine Handlung [am Patienten selbst] verübt wird, sondern lediglich ein Hindernis entfernt wird.» [7] Es scheint also aus jüdischer Sicht erlaubt zu sein, einem Patienten, der dem Tod sehr nahe ist ("goses») und nicht sterben kann, das Element, das ihn an seinem Tod hindert, zu entfernen, um so den Eintritt seines Todes zuzulassen. Eine andere Quelle, die in einem eher etwas weiteren $\mathrm{Zu}$ sammenhang mit der Sterbehilfe zu sehen ist, findet sich im Talmudtraktat Ketubot (104a): Dort wird berichtet, wie Rabbi Jehuda Hanassi (2. Jh. n. Chr.) von einer unheilbaren, entkräftenden Krankheit heimgesucht wurde. Ihm diente eine Bedienstete, die in der rabbinischen Literatur als eine Frau mit exemplarischer Frömmigkeit sowie moralischem Charakter beschrieben wird. Diese Bedienstete, so wird berichtet, habe für den Tod von Rabbi Jehuda Hanassi gebetet, damit dieser endlich von seinem Leiden erlöst werde. Gestützt auf diese Erzählung erklärte Rabbenu Nissim von Gerondi (13. Jh., Kommentar zu Nedarim 40a, eigene Übersetzung): «Meiner Meinung nach [...] gibt es Umstände, wo man für den Tod eines Kranken beten sollte, beispielsweise, wenn die kranke Person sehr leidet und ein Überleben nicht möglich ist, wie wir es im Fall der Bediensteten von Rabbi [Jehuda Hanassi] gesehen haben." Rabbiner Lord Jakobovits bezeichnete den Ausspruch von Rabbenu Nissim als grosse Konzession der "Sterbehilfe» gegenüber, denn im jüdischen Glauben spielen Gebete eine sehr wichtige Rolle und an ihrer Macht wird nicht gezweifelt.

\section{Aktuelle Haltung rabbinischer Autoritäten}

Die "moderne» Diskussion um die jüdische Haltung bezüglich Sterbehilfe wurde von Rabbiner Lord Jakobovits in seinem Pionierwerk "Jewish Medical Ethics" wie folgt zusammengefasst: "Jegliche Form von aktiver Sterbehilfe ist nach jüdischem Gesetz strengstens verboten. Sie gilt als Mord [...]. Gleichzeitig wird aber von der Halacha erlaubt - ja vielleicht sogar verlangt - einen Faktor zu entfernen, ob ausserhalb des Patienten oder am Patienten selbst [solange der Patient nicht bewegt wird], der künstlich den Sterbevorgang verlängern könnte.» [8] Lord Jakobovits betont, dass diese Regel lediglich im Falle eines "goses" Gültigkeit besitze. Somit ist auch die passive Sterbehilfe bei einem Patienten, der noch Wo- 
chen oder Monate zu leben hat, grundsätzlich verboten.

Rav Elieser Jehuda Waldenberg, speziell für medizinische Fragen eine angesehene rabbinische Autorität, behandelt in seinem medizinisch-halachischen Werk "Ziz Elieser» eingehend die Problematik der Sterbehilfe. Rav Waldenberg vertritt dabei die Auffassung, dass jeder Mensch nach jüdischem Gesetz verpflichtet sei, alles zu tun, was in seiner Macht stehe, um einem Sterbenden das Leben zu erhalten, auch wenn dies nur für kurze Zeit gelingen sollte. Dies gelte nach Rav Waldenberg auch dann, wenn der Sterbende schwer leide und den Wunsch anbringe, man möge seinen Tod beschleunigen [9]. Allerdings sei es gemäss jüdischem Gesetz erlaubt, dem Sterbenden narkotische Analgetika wie Morphium zu verabreichen, sogar wenn diese den Tod beschleunigen könnten, aber nie mit der Absicht, ihm das Leben $\mathrm{zu}$ verkürzen, sondern lediglich, um seine Schmerzen zu lindern [10]. Rav Waldenberg hält weiter fest, dass Bluttransfusionen, Sauerstoff, Antibiotika sowie orale oder parenterale Ernährung bei einem unheilbar kranken Patienten bis zu dessen Tod weiter gegeben werden müssten [11]. Rav Schlomo Salmen Auerbach, eine der grössten rabbinischen Autoritäten des 20. Jahrhunderts, betont ebenfalls, dass einem unheilbar und schwerstkranken Patienten Nahrung und Sauerstoff zugeführt werden müssten, und zwar sogar dann, wenn dies gegen seinen Willen geschehen sollte [12].

Rav Bleich hat die jüdische Haltung gegenüber der Sterbehilfe wie folgt beschrieben: "Das Praktizieren von Sterbehilfe, ob aktiv oder passiv, steht im Widerspruch zur Lehre des Judentums. Jegliche positive Handlung, die bezweckt, den Tod eines Patienten zu beschleunigen, ist nach jüdischem Gesetz ein Mord, auch dann, wenn der Tod nur um einige Momente beschleunigt wurde. Es spielt keine Rolle, wie ehrenwert die Motive der Person waren, die aus Wohlwollen Sterbehilfe geleistet hat, ihre Tat war ein Mord [...]. Nur der Schöpfer dieser Welt, der uns das Geschenk des Lebens gegeben hat, hat das Recht, dieses Leben wieder zu nehmen, auch dann, wenn das Leben eher zur Last denn zum Segen geworden ist.» [5] Rav Bleich betont im weiteren den Spezialfall "goses". Auch hier gelte, dass der Tod nicht aktiv beschleunigt werden dürfe, aber unter gewissen Umständen könne man es bei einem "goses» unterlassen, überhaupt mit einer Behandlung zu beginnen. Rav Bleich zitiert einige Autoritäten, die nicht nur das Unterlassen einer Behandlung zulassen, sondern sogar die Auffassung vertreten, dass jede Handlung, die den Sterbeprozess eines "goses" verlängert, verboten sei. So schreibt beispielsweise Rav Mosche Feinstein in seinen Responsen: "Es ist sicherlich verboten, $\mathrm{zu}$ versuchen, das Leben einer sterbenden Person zu verlängern, wenn dies in [zusätzlichem] Schmerz und Leiden resultieren würde. Auch das Leben zu verkürzen [...] ist verboten. Wenn jemand eine solche Handlung begeht [...], auch wenn es auf Geheiss des Patienten geschieht, so ist er ein Mörder [...].» [13] Prof. Abraham, zeitgenössischer Experte in medizinisch-halachischen
Fragen, hat den Versuch unternommen, Richtlinien im Zusammenhang mit der Problematik der Sterbehilfe aus jüdischer Sicht aufzustellen [14], die im folgenden kurz zusammengefasst sein sollen:

1. Grundsätzlich müssen alle Patienten - unabhängig von ihrem Zustand - mit Nahrung, Flüssigkeit, Sauerstoff und anderen lebenserhaltenden Elementen versorgt werden, und zwar auch dann, wenn diese lebenserhaltenden Elemente auf unübliche Weise verabreicht werden müssen.

2. Patienten mit schwersten chronischen Krankheiten, die nicht terminal sind, müssen genau gleich wie alle anderen Patienten behandelt werden.

3. Patienten mit terminalen Krankheiten, die am Sterben sind ("goses»), müssen grundsätzlich ebenfalls wie alle anderen Patienten behandelt werden. Wenn ein "goses" nun aber beispielsweise einen Herz- oder Atmungsstillstand erleidet oder andere schwere Komplikationen auftreten, die einer grösseren Behandlung bedürften, so dass sich die Leiden des Sterbenden noch verschlimmern könnten, gilt folgendes:

a) wenn der Herz- oder Atemstillstand durch die terminale Krankheit bedingt ist, d.h. im Rahmen des unvermeidlichen Krankheitsverlaufs erwartet werden konnte, dann muss eine Reanimation grundsätzlich nicht unbedingt versucht werden, vielleicht wäre es sogar ein Fehler, eine solche zu versuchen;

b) wenn aber der Herz- oder Atemstillstand oder andere Komplikationen unerwartet und unabhängig von der terminalen Krankheit auftreten, dann muss auch bei einem "goses» eine vollständige Behandlung eingeleitet werden, wie dies für jeden anderen Patienten auch geschehen würde. Diese Grundregel gilt aber nur in denjenigen Fällen, in welchen die Leiden und der Todeskampf des Sterbenden dadurch nicht verstärkt werden.

\section{Konsequenzen für die aktuelle Diskussion}

In der Beurteilung der Debatte um die Legalisierung der aktiven Sterbehilfe respektive des ärztlich-assistierten Suizides wird klar, dass die Befürworter einer solchen Regelung mit ihren Argumenten eindeutig zentralsten Grundprinzipien der jüdischen Medizinethik widersprechen. Besonders hervorzuheben sind die Missachtung des biblischen Tötungsverbotes sowie des Prinzips, das Leben als Leihgabe $\mathrm{zu}$ betrachten. Gerade dieses Konzept der "Leihgabe» menschlichen Lebens scheint generell im Gegensatz zum aktuellen Trend der säkularen Ethik zu stehen, bei welcher der Autonomie des Patienten immer höheren Stellenwert eingeräumt wird. Es handelt sich aber um einen schwerwiegenden Irrtum, anzunehmen, dass die Entscheidung eines Individuums, sich umzubringen, nur dieses selbst und nicht die Gesellschaft als Ganzes betrifft. Verschiedene Untersuchungen im Zusammenhang mit der Legalisierung des ärztlich-assistierten Suizids haben beispielsweise gezeigt, dass vor allem den alten und armen Men- 
schen, die sich ohnehin bereits am Rande der Gesellschaft befinden, am häufigsten das «Recht zu Sterben" zugebilligt beziehungsweise nahegelegt würde. Der moralische Druck auf diejenigen, die trotz ihrer Leiden und trotz ihrer «kostenverursachender Wirkung" weiterleben möchten, würde gemäss diesen Studien zweifelsohne steigen. Es stellt sich auch die Frage, wem durch eine Legalisierung der aktiven Sterbehilfe beziehungsweise des ärztlich-assistierten Suizids letztendlich tatsächlich geholfen würde: Dem leidenden Individuum, welches unsere dringende emotionale Unterstützung benötigt, oder uns selbst als Gesellschaft, welche die Probleme der stetig steigenden Gesundheitskosten und der limitierten Ressourcen zu lösen versucht und sich dabei der "unbequemen" Patienten auf "einfache" Art und Weise entledigen könnte? Die oben angesprochene Missachtung des Tötungsverbotes wird übrigens keinesfalls nur von jüdischer Seite kritisiert: «Die Entscheidung des niederländischen Parlaments, das Tötungsverbot in bestimmten Fällen aufzuheben und ärztlich gestützte Euthanasie zuzulassen, rührt an den Grundfesten einer humanen Gesellschaft», beurteilte beispielsweise der Präsident der Bundesärztekammer in Deutschland, Prof. Jörg-Dietrich Hoppe, die Entwicklung in Holland. Die bisher gemachten Erfahrungen dort lehren uns denn auch, dass die Legalisierung der aktiven Sterbehilfe mit vielen Problemen verbunden ist und mehr als bedenkliche Konsequenzen mit sich bringt: Die Ausweitung der Regelung auch auf behinderte Neugeborene und Kinder sowie auf Patienten mit psychischen Krankheiten ist nur ein Beispiel. Zudem wurden allein in den letzten Jahren Tausende Fälle von "aktiver Sterbehilfe» bekannt, welche ohne den ausdrücklichen Wunsch von Patienten durchgeführt wurden. Von einigen niederländischen Politikern wird gar eine ausdrückliche Meinungsäusserung der Patienten gefordert, falls diese nicht aktive Sterbehilfe wünschten. Die Entwicklung in Holland lässt also schlimmste Befürchtungen wahr werden: «Tötung auf Verlangen" wird, wie die Juristin Birgit Reuter in einer kürzlich erschienen Doktorarbeit belegt [15], von niederländischen Ärzten nicht nur an Kranken vollzogen, die zu Willensäusserungen nicht fähig sind, sondern zum Teil sogar an Entscheidungsfähi- gen, die man gar nicht erst fragt. Wohin wird uns diese Entwicklung führen? Sollten wir aus den in Holland gemachten Erfahrungen nicht unsere Lehren ziehen? Aus Sicht der jüdischen Medizinethik ist klar, dass grundlegende Prinzipien wie die Unantastbarkeit und Heiligkeit menschlichen Lebens sowie das biblische Verbot der Tötung und der Selbsttötung auf keinen Fall aufgegeben werden dürfen. Die Geschichte zeigt uns, dass das Überleben einer Zivilisation auch von der Stärke ihrer moralischen Wertvorstellungen abhängt. Unsere moderne Gesellschaft, mit all ihren Fortschritten in Wissenschaft, Medizin und Technologie, scheint sich auf den gefährlichen Weg zu begeben, grundlegende ethische Wertvorstellungen zu missachten. Es lässt sich deshalb nur hoffen, dass Argumente wie diejenigen der jüdischen Medizinethik nicht ungehört verhallen!

\section{Literatur}

1 ben Maimom (Maimonides) M. Mischneh Tora, Rotseach, 2:2 2 Schostak Z. Is there patient autonomy in halacha? ASSIA, A journal of jewish medical ethics 1995;2,2:22-7.

3 Jakobovits I. Medical experimentation on humans in jewish law. In: Rosner F, Bleich JD (eds.). Jewish bioethics. New York: Hebrew Publishing; 1979. p. 377-83.

4 Leibowitz J, Shashar M. Gespräche über Gott und die Welt. Frankfurt am Main/Leipzig: Insel; 1994.

5 Bleich JD. Judaism and healing. New York: Ktav; 1981.

6 ben Samuel J. Sefer Hachassidim, Nr. 273.

7 Isserles M. Kommentar zum Schulchan Aruch, Joreh Deah, 339:1.

8 Jakobovits I. Jewish medical ethics. New York: Bloch Publishing; 1975.

9 Waldenberg EJ. Ziz Elieser, V, 28:5, 29; IX, 47:5; X, 25:6.

10 Waldenberg EJ. Ziz Elieser, XIII, 87.

11 Waldenberg EJ. Ziz Elieser, XIV, 80.

12 Auerbach SS. In: Halacha Urefuah 2 (1981):131.

13 Feinstein M. Iggerot Mosche, Joreh Deah, 2, Nr. 174.

14 Abraham AS. Euthanasia. ASSIA, A journal of jewish medical ethics $1989 ; 1,2: 36-9$.

15 Reuter B. Die gesetzliche Regelung der aktiven ärztlichen Sterbehilfe des Königreichs der Niederlande - ein Modell für die Bundesrepublik Deutschland? Frankfurt am Main: Peter Lang; 2001.

In Yves Nordmanns Buch «Zwischen Leben und Tod - Aspekte der jüdischen Medizinethik», Peter Lang Verlag, 1999/2000, finden sich weiterreichende Ausführungen sowie zahlreiche zusätzliche Literaturangaben. 